\title{
Phytotoxicity and Biotransformation of Fenbendazole in Ribwort
}

\author{
Barbora Szotáková ${ }^{1}$, Lucie Raisová Stuchlíková ${ }^{1}$, Lenka Skálová ${ }^{1}$, Radka Podlipná ${ }^{2}$ \\ ${ }^{1}$ Department of Biochemical Sciences, Faculty of Pharmacy in Hradec Králové, Charles University \\ Akademika Heyrovského 1203, Hradec Králové, Czech Republic \\ ${ }^{2}$ Laboratory of Plant Biotechnologies, Institute of Experimental Botany, Czech Academy of Sciences \\ Rozvojová 263, Praha 6, Czech Republic \\ szotakova@faf.cuni.cz; podlipna@ueb.cas.cz
}

\section{Extended Abstract}

Fenbendazole (FEN) is anthelmintic drug often used in pig and poultry management. Usefulness of anthelmintic drugs is uncontested, but at the same time they represent a risk to ecosystems. Anthelmintics administered to animals enter into environment primarily through their excretion in faeces or urine. Following excretion, these substances may persist in the environment and impact non-target organisms. Ecological hazards of benzimidazole anthelmintics in aquatic ecosystems have been studied with results documenting acute as well as chronic toxicity against the freshwater invertebrate Daphnia magna, with FBZ being the most toxic [1]. Plants come into contact with anthelmintics and other veterinary drugs in pastures. But in contrast to the situation regarding animals, information about the toxicity and fate of anthelmintics in plants is very limited [2].

The present study was designed to test phytotoxicity and biotransformation of the benzimidazole anthelmintic FBZ in the ribwort plantain (Plantago lancelota). This common meadow plant is exposed to anthelmintics from the excrements of treated animals. The uptake and biotransformation of FBZ was studied and occurrence of FBZ and its metabolites in cell suspensions, roots, basal parts of the leaves and tops of the leaves were followed up. Overproduction of proline is a widespread response observed in plants experiencing various stresses. The quantification of this amino acid is therefore very useful to assess the physiological status and more generally to understand stress tolerance in plants.

The concentration of proline in plantain leaves of control and FBZ treated plants was measured and significant increase of proline concentration was observed after FBZ treatment. UHPLC-MS/MS analysis led to the identification of 14 metabolites of FBZ formed in the ribwort. In both model systems, the same types of FBZ biotransformation reactions were found, but the spectrum and abundance of the FBZ metabolites detected in cell suspensions and regenerants differed significantly. Most of these metabolites can be considered deactivation products, but a substantial part of them may readily be decomposed to biologically active substances which could negatively affect ecosystems. In conclusion, FBZ in ribwort induced stress and underwent various biotransformations. The scheme of FBZ metabolic pathway in ribwort has been proposed.

This project was supported by Czech Science Foundation, grant No. 15-05325S.

\section{References}

[1] S. J. Oh, J. Park, M. J. Lee, S. Y. Park, J. H. Lee, K. Choi, "Ecological hazard assessment of major veterinary benzimidazoles: acute and chronic toxicities to aquatic microbes and invertebrates," Environ. Toxicol. Chem., vol. 25, pp. 2221e2226, 2006.

[2] L. Stuchlíková, R. Jirásko, L. Skálová, F. Pavlík, B. Szotáková, M. Holčapek, R. Podlipná, "Metabolic pathways of benzimidazole anthelmintics in harebell (Campanula rotundifolia)," Chemosphere, vol. 157, pp. 10-17, 2016. 